\title{
El comportamiento criminal desde un punto de vista evolucionista
}

\author{
José E. García \\ Universidad Católica \\ Asunción, Paraguay
}

Recibido: 8 de mayo de 2015 / Aprobado: 13 de julio de 2015

Las teorías sobre las causas del comportamiento criminal y sus variantes fascinaron la atención de los investigadores, al menos desde mediados del siglo XVIII. En nuestros dias, la psicología evolucionista produjo algunos aportes importantes para entender los crímenes y homicidios como formas de adaptación humana a los desafíos generados en situaciones ambientales especificas, a través de lo que se denomina mecanismos psicológicos evolucionados. Este artículo examina brevemente los antecedentes históricos del estudio biológico del comportamiento criminal y los desarrollos actuales surgidos en la psicología evolucionista. El enfoque es teórico, con una revisión de las fuentes publicadas más relevantes.

crimen / homicidio / psicología evolucionista /mecanismos psicológicos evolucionados

\section{Criminal Behavior from an Evolutionary Point of View}

Theories concerning the causes of criminal behavior and its variants has fascinated researchers at least since the mid-eighteenth century. Nowadays, evolutionary psychology had made important contributions to the understanding of the crimes and murders as forms of human adaptation to the challenges posed by specific environmental situations, through what now is called evolved psychological mechanisms. This article briefly reviews the historical background of the biological study of criminal behavior and the current developments that emerged in evolutionary psychology. The approach is theoretical, with a review of the most relevant published sources.

crime / homicide / evolutionary psychology / evolved psychological mechanisms

Correo electrónico: joseemiliogarcia@hotmail.com 


\section{El CONTEXTO BÁSICO DE LA EVOLUCIÓN Y LA PSICOLOGÍA}

La publicación por Charles Darwin (1809-1882) de la teoría de la evolución por vías de la selección natural es considerada un acontecimiento fundamental para las ciencias biológicas y el estudio de los procesos de cambio morfológico que operan en los seres vivos (Shanahan, 2004). Conceptos como el de la adaptación y el cambio evolutivo, así como el constructo explicativo básico de la selección natural pasaron a ocupar un lugar central en la teorización de ciencias como la biología del desarrollo (Hall, 1999), la embriología (Raff, 1996) y la anatomía (Passingham y Wise, 2012). Aunque al formularse -en la segunda mitad del siglo XIX- la teoría ya había dado lugar a controversias acaloradas, generalmente motivadas por resistencias nacidas en los dogmas del conservadurismo religioso, los debates encontraron su despliegue en muy variados escenarios, desde la geología a la política (Ruse, 2001). Hoy, el hecho de la evolución es aceptado por una vasta mayoría de los científicos (Stefoff, 1996) y cada especialidad biológica se halla informada y enriquecida por los conocimientos logrados en este campo. En general, los biólogos consideran el proceso evolutivo como un fenómeno comprobado y fuera de discusión, una de las grandes formulaciones logradas en el ámbito de la ciencia, tan demostrable en sus efectos como la de que la tierra se encuentra en órbita alrededor del sol (Sapp, 2003).
Las implicaciones de la teoría sobre el ámbito propio de la psicología también fueron evidentes desde el principio (Richards, 1989) y generaron muchas explicaciones del funcionamiento mental centradas en las nociones que consideraban los cambios en las formas vivientes como producto de secuencias de adaptación muy lentas. Tooby y Cosmides (2005) señalan que pese a la relativa marginación que afectó al darwinismo en el interior de la psicología durante la mayor parte del siglo $\mathrm{XX}$, unos pocos autores se esforzaron por desarrollar un pensamiento que buscaba trasladar los conocimientos del proceso evolutivo a los problemas centrales de la mente y el comportamiento. Desde luego, no podía ser de otra manera. La selección natural establece que los cambios producidos en el ambiente, que también pueden conceptualizarse como los nichos ecológicos de las diferentes formas taxonómicas (Alcock, 1989), generan una fuerte presión adaptativa sobre los organismos, obligándolos a modificar su forma física en respuesta a tres funciones básicas de supervivencia: a) la necesidad de reproducción, es decir, la transmisión de los genes de una generación a la siguiente, b) la conducta de forrajeo, esto es, la incorporación regular del alimento, y c) la búsqueda de refugio para guarecerse de los elementos y precaver cualquier daño a la integridad corporal. Si el animal fracasa en cumplir cualquiera de estas funciones básicas, sus destinos no son muy alentadores, re- 
duciéndose a la muerte como individuo o la extinción como especie.

Darwin publicó su influyente obra El origen de las especies en 1859, con el propósito de establecer el mecanismo elemental que define a la selección natural (Darwin, 1859). Este libro no debe apreciarse únicamente como la exposición convencional de una teoría científica, sino además como una de las obras fundamentales que ha legado la ciencia moderna (Francis, 2007). Pero años más tarde, en 1871, Darwin también daba a conocer una segunda contribución, que en la lengua española llegó a ser comúnmente conocida como El origen del hombre pero que en realidad debió ser La ascendencia del hombre, si hemos de atenernos estrictamente a la correcta traducción de su título original (Darwin, 1871). Esta obra fijó la agenda para el estudio de la evolución humana al seguir los mismos principios que habían sido aplicados previamente al comportamiento animal. Aún aparecería otro trabajo de gran importancia al año siguiente, llamado La expresión de las emociones en el hombre y los animales (Darwin, 1872). Como hemos dicho, El origen del hombre abrió el camino para el estudio de la especie humana desde una perspectiva evolucionista, pero en la obra de 1872 se dieron algunos pasos adelante al aplicar persuasivamente el siguiente razonamiento: tan importante como la forma física para la supervivencia del individuo son los hábitos comportamentales característicos de la especie. De esta manera, morfología y comportamiento se situaban en una relación de igualdad y eran presentados como inextricablemente unidos. La conducta y los hábitos del animal determinaban también la eficacia biológica, la adaptación al medio y el éxito reproductivo potencial. De hecho, numerosos investigadores en el ámbito de las ciencias del comportamiento tomaron suficientemente en serio los postulados que había adelantado Darwin, propugnando formas variadas y a veces divergentes de someter asuntos concernientes a la mente y el comportamiento a los enunciados rectores que ofrecía el nuevo enfoque.

Aún en vida de Darwin algunos naturalistas amigos suyos, como George John Romanes (1848-1894), se dispusieron a extender estos axiomas a la investigación del comportamiento animal, comenzando así la psicología comparada y generando, a su vez, el uso del controvertido método anecdótico (Romanes, 1884). En sus estudios, Romanes aceptó la triple división de Darwin en cuanto a la acción refleja, el instinto y la inteligencia o razón, que debían estimarse como expresión de una secuencia evolutiva (Tolman, 1987). Pero el método anecdótico no fue bien recibido y pronto resultó completamente arrinconado hacia la periferia de la psicología por el también evolucionista Conwy Lloyd Morgan (1852-1936). Este desbancó cualquier intento de fundamentarse en anécdotas con la aplicación rigurosa de su famoso canon (Morgan, 1896). En Estados Uni- 
dos, William James también demostró una fuerte absorción intelectual de la perspectiva evolucionista en sus célebres Principles of Psychology (James, 1890) y lo mismo puede afirmarse de los seguidores de la escuela funcionalista, sustancialmente inspirada en las ideas de James (Shiraev, 2014). El funcionalismo estuvo enfocado en el modo general como funciona la mente y en cómo los organismos la utilizan para adaptarse a su entorno ambiental (Schultz y Schultz, 2011). Ellos buscaron entender la función de los procesos mentales en lugar de proveer una descripción estática de sus contenidos (Hergenhahn y Henley, 2013).

Entre los funcionalistas destacó el trabajo de James Mark Baldwin (18611934) con su énfasis sobre el desarrollo humano (Baldwin, 1895) y de lo que él denominó selección orgánica, un concepto que asignaba una importancia fundamental al fenómeno del aprendizaje como integrante del proceso de la evolución. En varios sentidos importantes Baldwin antecedió al trabajo, más universalmente conocido en la actualidad, de Jean Piaget (1896-1980) (Cahan, 1984). A comienzos del siglo XX, el psicólogo William McDougall (1871-1938) elaboró una psicología social que acusó un fuerte acento evolucionista y otorgó una gran importancia al estudio del comportamiento instintivo (McDougall, 1912). Sin embargo, no fue el primero. En el capítulo 24 de los Principles... -como señaló Röell (2000)-, James (1890) también hizo menciones especí- ficas a los instintos y los definió como “... la facultad de actuar de tal manera a producir ciertos fines, sin previsión de la finalidad, y sin educación previa para el desempeño" (p. 383). Como ellos otros autores incorporaron, con variados grados de profundidad, los preceptos darwinianos al contexto de la teorización psicológica, desde el psicoanálisis de Sigmund Freud (1856-1939) hasta el condicionamiento operante de B. F. Skinner (1904-1990).

En la década de 1970 tuvo especial importancia la sociobiología creada por el entomólogo estadounidense E. O. Wilson (1929-), que se propuso estudiar la conducta social descubriendo sus bases genéticas (Wilson, 2000). A mediados de la década de 1980 los conceptos se redefinieron un poco cuando irrumpió la psicología evolucionista, de gran influencia actual y a la que habremos de hacer varias referencias posteriores. La agenda de la psicología evolucionista es amplia y abarcadora, incluyendo numerosos problemas de interés directo para los psicólogos. Aunque comparten muchas ideas comunes, los psicólogos evolucionistas discrepan de los sociobiólogos en algunos aspectos críticos como el rol atribuido a la eficacia biológica. Para la sociobiología esta es una fuerza ciega que empuja a los seres humanos a maximizar el potencial de sus genes en la distribución poblacional, mientras los psicólogos evolucionistas ponen mayor énfasis en los mecanismos psicológicos evolucionados, que implican la existen- 
cia de una arquitectura del cerebro con funciones de dominio específico. La psicología evolucionista, al mismo tiempo, asimiló los conceptos originados en la revolución cognitiva a sus modelos y propuestas de investigación (Wortley, 2011). Teniendo en cuenta esta continua diversificación de los temas y focos de interés, en este artículo no perseguimos la vana intención de analizar todas las facetas actualmente desarrolladas sino solo uno de los campos en los que el estudio darwiniano del comportamiento ha comenzado a fructificar en años recientes, como es la investigación del comportamiento criminal. El artículo es teórico y argumentativo y su estrategia de corte documental. La revisión está centrada en los fundamentos históricos, así como en una exposición sumaria de los avances actuales. En la siguiente sección nos detendremos brevemente en los argumentos principales que responden al problema.

\section{LA APROXIMACIÓN CLÁSICA}

La disciplina que se ocupa de las causas del crimen es la criminología. Pero definir este campo no siempre resulta sencillo. Es común que el foco esté puesto en el comportamiento infractor a la ley y no necesariamente en la conducta criminal en cuanto tal (Walklate, 2007). Las discordancias también surgen cuando se utiliza un criterio histórico. Si conceptualizamos a la criminología como la ciencia que persigue el estudio científico del crimen y los criminales, la discusión se vuelve compleja por la abundancia de tradiciones teóricas divergentes (Renneville, 2006). Cada una de ellas presume alguna clase particular de ortodoxia y genuina validez científica. En el caso de la criminología francesa, los comienzos pueden atribuirse a Bénédict Augustine-Morel (1809-1873) o a Prosper Lucas (18081885) (Vallejo, 2009) si se entiende como el estudio de la psicología criminal, o a Emile Durkheim (1858-1917) y André-Michel Guerry (1802-1866) si es considerada como la sociología de la desviación. Finalmente, si es el conocimiento antropológico de los criminales, la atención se dirige a Cesare Lombroso (1836-1909). Queda claro que la determinación precisa sobre los orígenes es en gran medida una cuestión de criterios. Desde el principio de estos estudios quedó marcada una bifurcación en teorías sobre los fundamentos biológicos o genéticos y las orientaciones de carácter más social. Y aunque pueda sonar como una opinión demasiado excluyente, quienes apoyan el primer tipo de explicaciones a menudo consideran a las segundas como un simple ruido distractor respecto a las verdaderas causas que fomentan la conducta criminal.

En una discusión amplia del tema, Figueredo, Gladden y Hohman (2012) clasificaron los enfoques existentes para el estudio del comportamiento criminal en dos tendencias principales. Por una parte agruparon las teorías del modelo estándar de las ciencias sociales que 
incluyen, a saber: a) teorías clásicas, b) teorías positivistas, c) teorías funcionalistas, d) teorías del aprendizaje social, cultural y subcultural, e) teorías del control, f) teorías cognitivas y g) teorías tradicionales de la personalidad. Del otro lado están las aproximaciones de la ciencia social evolucionista, que abarcan: a) teorías de la genética comportamental, b) teorías epigenéticas y de la heredabilidad reactiva, c) teorías de la selección sexual, d) teorías sobre la inversión parental diferencial, e) teorías de la desventaja competitiva, f) teoría de la selección dependiente de la frecuencia, g) teoría del estrés patógeno y h) teorías sobre historia de la vida. Cabe señalar que la expresión modelo estándar de las ciencias sociales fue introducida en un capítulo de Tooby y Cosmides (1992) para aludir a un punto de vista según el cual los determinantes críticos para la formación del comportamiento humano son los que impone la cultura en que se socializa el individuo y que estos patrones son transmitidos por los grupos de pertenencia, con casi total exclusión de otras causas hereditarias. Consideran que el artífice principal de este pensamiento es el sociólogo francés Emile Durkheim (1858-1917), en especial por las ideas expuestas en sus famosas Reglas del método sociológico, publicadas originalmente en 1894 (Durkheim, 1919).

La suposición de que existe alguna relación entre el comportamiento criminal y algún sustrato particular que involucra la anatomía humana, especialmente el cerebro, ha sido objeto de especulación desde hace largo tiempo. Numerosos son los investigadores que se mostraron interesados en los diversos aspectos que encierra este problema y generaron hipótesis y teorías bien articuladas ya desde el siglo XVIII por lo menos (Rafter, 2008). Creador de la popular escuela de la fisiognomía en esa centuria, el suizo Johann Kaspar Lavater (1741-1801) creía que el carácter de una persona podía determinarse a través de las mediciones del rostro. Por ejemplo, una nariz arrogante, unos ojos furtivos y un mentón débil denotaban un carácter criminal (Sutherland, Cressey y Luckenbill, 1992). Con los inicios de la frenología unas décadas más tarde (Renneville, 2000), la aproximación neuroanatómica liderada por Franz Joseph Gall (1758-1828) y que abrió el campo para investigar la localización de funciones psicológicas diversas en la masa encefálica, el cerebro de los criminales se convirtió en el objeto central de estudio. La suposición central de la frenología fue que cada sección del cráneo, y por consiguiente del cerebro, era responsable por un aspecto diferente del funcionamiento mental humano (Padhy, 2006).

Por su parte, Gall pensaba que unas pequeñas elevaciones en la superficie del cráneo, susceptibles de palparse con el tacto y que eran denominadas por él protuberancias, indicaban una formación correspondiente que habría 
de darse en la estructura congénita del cerebro. Allí donde se detectaba esa prominencia podía denotarse también una actividad psicológica sobresaliente. Dentro del mapeo que hizo Gall de todas esas zonas del cerebro y a las que organizó inicialmente en veintisiete facultades que su asistente Johann Gaspar Spurzheim (1776-1832) aumentó a treinta y cinco -en concreto, veintiún afectivas y catorce intelectuales- (Haskins, 1839), se pensó que las propensidades inferiores como la combatividad, el secretismo y la codicia son las que se encuentran asociadas al crimen (Sutherland, Cressey y Luckenbill, 1992). La frenología fue sucedida por una antropología criminal en el siglo XVIII, con fuerte influencia de las ideas evolucionistas. El célebre criminólogo italiano Cesare Lombroso, que escribió y reflexionó extensamente sobre los fundamentos biológicos del comportamiento criminal, fue otro eslabón crítico en la atribución de este a causas biológicas. Su libro, titulado $E l$ hombre delincuente (Lombroso, 1876), fue muy leído e influyente. A través de esta obra y otras muy importantes, fundó una psicobiología del delito, en el que este respondía a causas innatas, físicas y biológicas. En las cárceles, auténticos laboratorios de la conducta antisocial, aquellos nacidos para ser criminales podían identificarse por la apariencia física y la capacidad cerebral que exhibían los prisioneros (Anthony y Anthony, 2008). Pero desde la publicación de las grandes obras de Darwin, sus principios establecieron el marco de fondo para una inserción amplia de la criminología en el escenario moderno de la ciencia. Brown, Esbensen y Geis (2013) creen que la visión del comportamiento de los criminales como un atavismo, esto es, un pretendido retroceso hacia un estadio anterior y más primitivo del ser humano conforme al modelo que defendió Lombroso, es una deuda intelectual directa con Darwin.

\section{LA PSICOLOGÍA EVOLUCIONISTA}

Frente a la considerable amplitud que revisten estos problemas, los psicólogos no tardaron en comprender la importancia derivada del nuevo enfoque. Con diversos grados de profundidad, la psicología incorporó las nociones darwinianas a una variedad de tópicos. El estudio del comportamiento criminal ha sido uno de ellos. Desde luego, los intereses en la comprensión de las singularidades que son privativas de la mente homicida no comenzaron ni tampoco acabarán con los aportes de los evolucionistas. La investigación avanzó a todo lo largo del siglo $\mathrm{XX}$, en directa conexión con los diferentes enfoques teóricos en las ciencias del comportamiento. Desde mediados de la década de 1980 se ha ido conformando el campo de la psicología evolucionista, que es la más reciente forma de aplicación de los principios de la selección natural y el estudio de la adaptación al dominio del comportamiento. Sus áreas de exploración crecieron con inusitada rapidez, abarcando desde los 
procesos involucrados en la evolución de la cognición y el lenguaje hasta la conducta violenta y las peculiaridades de la sexualidad humana, entre otros muchos asuntos. En fecha reciente comenzó a tomar forma la psicología forense evolucionista o la exploración que se produce en la intersección justa entre la psicología y la ley (Duntley y Shackelford, 2008). Como ocurre con el resto de esta psicología, la teoría de la evolución constituye el marco de referencia metateórico que unifica el grueso de la investigación. Así como los procesos evolutivos sirvieron para moldear las adaptaciones psicológicas con la aparición de funciones orientadas a la solución de problemas específicos y la formación de respuestas efectivas a los problemas planteados por la supervivencia y la reproducción, también parece haber actuado en relación a comportamientos que son, en apariencia, desadaptativos. En este punto se debe mencionar que un principio central de la psicología evolucionista es que la criminalidad no solo se hereda, como lo hace cualquier otra característica física o psicológica, sino que también es mantenida por acción de la selección natural. De esta forma, la predisposición al comportamiento criminal podría encontrarse distribuida normalmente en la población, habitando en todos los individuos de alguna manera. Se ha supuesto inclusive que todos los seres humanos están igualmente predispuestos a ser criminales (Kinner, 2003).
Esta misma lógica es muy efectiva para el análisis de los problemas que atañen al estudio de los temas comunes en la psicología forense. Se reconoce así que determinados crímenes o hechos violentos como el asesinato, la violencia no letal, la violación y el hurto son manifestaciones de problemas evolutivos recurrentes entre los individuos, que aparecen una y otra vez a lo largo de nuestra historia como especie. Pero como no todos ellos son iguales en su recurso a la violencia y al grado y extensión de sus efectos, una cuestión fundamental habrá de ser cuáles deberían considerarse como necesarios de una explicación. Por ejemplo, Duntley y Shackelford (2008) se ocupan de cuestiones clave como homicidio, violencia en la pareja, acoso sexual, violación, prostitución, asunción de comportamientos de riesgo, hurto y psicopatía. Otras incógnitas, como las derivadas de la incidencia de la pedofilia, también caben dentro de esta compleja gama de intereses (Quinsey, 2010) y pueden abordarse con éxito desde esta perspectiva. Pero si estos problemas son tan antiguos y perdurables en toda la historia humana es porque su existencia responde a determinadas adaptaciones evolutivas, de lo contrario no se hallarían presentes a lo largo de un tiempo tan dilatado. Su explicación, por lo tanto, es un verdadero desafío para los psicólogos y es por eso que varios avanzaron hipótesis y esquemas para categorizarlos.

Los humanos hemos desarrollado adaptaciones situacionales complejas 
para infligir costos a otros seres humanos y de esta manera resolver un gran número de problemas para la supervivencia (Buss y Duntley, 2006). Entre ellos figuran el apropiarse de los recursos que pertenecen a otros, defenderse contra los ataques, confrontar a rivales intrasexuales, ascender en las jerarquías de dominancia, disuadir a las parejas románticas de incurrir en infidelidades, recuperar relaciones afectivas perdidas y obtener acceso sexual a parejas que de otro modo serían inaccesibles. Duntley y Buss (2012) estudiaron la forma en que comportamientos como el acecho, que incluye la intrusión obsesiva en las relaciones con miembros del otro sexo, la persistencia en el cortejo y el acoso criminal pueden analizarse como estrategias para resolver problemas de apareamiento. Los datos de la investigación intercultural demuestran que problemas reiterativos como el robo, la agresión de varón a varón, la violencia en la pareja, los atracos sexuales, el acoso y el asesinato eran tan comunes en las culturas primitivas como lo son en las actuales (Buss, 2012). En un importante artículo, McKibbin, Shackelford, Goetz y Starratt (2008) discuten la evidencia de que los hombres puedan haber desarrollado mecanismos psicológicos evolucionados que los inclinen hacia la violación cuando ocurre en contextos específicos. Para que estas adaptaciones se fijaran duraderamente como patrones cognitivos habría sido necesario que el comportamiento violador produjese beneficios que sobrepasaran los costos que implica entrar en una relación de este tipo. Uno de ellos es la evaluación del atractivo sexual de las potenciales víctimas de violación. La mujer está en el pináculo de su capacidad reproductiva en torno a los veinticinco años, la edad que es precisamente la más frecuente en las violaciones consumadas. Si la producción de la pregnancia puede garantizarse mediante una copulación forzada de una sola vez, entonces la inclinación del violador sería asegurar el éxito mediante la elección de una mujer en condiciones de mayor fertilidad que otra en situación de fertilidad disminuida, ya sea a causa de la salud, la edad o por los motivos que fueran. Los hechos, en general, parecen ir de acuerdo con estos asertos.

Una comprensión acabada de los fundamentos reales que genera la ocurrencia de eventos como los mencionados podrán llevarnos hacia formas más efectivas y realistas de prevenir su incidencia destructiva en el futuro. Los psicólogos Martin Daly y Margo Wilson estuvieron entre los primeros en impulsar una línea de investigación muy consistente, que sirve para aclarar muchos de estos temas. Entre otras cosas, su trabajo condujo a una formulación novedosa de lo que se ha denominado el Efecto Cenicienta, basado en la trama argumental del célebre cuento infantil, para explicar las diferencias encontradas en el maltrato de los niños por parte de los padrastros en comparación con los progenitores biológicos. Los autores sostenían que debido a lo prolongado y costoso de la infancia humana, resulta 
muy improbable que los padres actúen de manera indiscriminada con todos los niños, es decir atendiendo a todos por igual, al momento de la crianza. Por el contrario, ellos se ven compelidos a ser discriminativos y eso los conduce a privilegiar a sus propios hijos en posible desmedro de aquellos que les son ajenos genéticamente. Esta diferencia se comprende mejor en función a lo que establece la teoría de la inversión parental, prediciendo que la proporción relativa de inversión de los progenitores, es decir el tiempo y energía dedicada al cuidado de la descendencia individual y que se realiza a expensas de los otros hijos, varía significativamente de una especie a otra y tampoco es igual para ambos sexos (Schmitt, 2010).

La inversión paternal se mantiene a costa de algunos sacrificios, distribuidos entre los beneficios a los niños, la certeza sobre la paternidad y las oportunidades que se pierden para nuevos apareamientos (Geary, 2005). Los progenitores que invierten su tiempo, recursos y energía en niños con los que no se encuentran genéticamente relacionados enfrentan una dispersión muy importante de recursos, no solo económicos sino también afectivos. A la larga, estos resultan inconducentes, pues no habrán de culminar con un resultado reproductivo específico. En esencia, ser padre adoptivo es cuidar infantes con genes extraños que no perpetuarán nuestras propias características sino las de otros hombres. Los niños en familias de este tipo son heridos o muertos en una abrumadora mayoría en comparación con los que crecen en compañía de sus dos padres biológicos (Cartwright, 2000). No es extraño que la violencia contra ellos sea la norma. Un modo extremo en la manifestación de este problema es el infanticidio. Muchos padres llegan a matar a los niños, pero ello se da en un grado considerablemente mayor cuando no existen lazos de sangre.

Mucha de esta lógica es igualmente aplicable a los individuos que asumen riesgos. Como pocas, esta es una variable sensiblemente relacionada con el sexo. Los hombres mueren más en accidentes de tránsito que las mujeres y también figuran con mayor frecuencia como actores de altercados agresivos (Daly y Wilson, 2001). El incurrir en conductas riesgosas tampoco es un problema que se presente de manera aislada. Con acertado criterio, Mishra y Lalumière (2008) las analizan junto con la conducta antisocial porque, en su opinión, guardan muchas características en común. Ambas involucran impulsividad, imprudencia, recompensa inmediata y comportamiento egoísta. También parece ofrecer una mejor adaptación funcional en determinados periodos de la vida, por ejemplo en la adolescencia y la adultez temprana debido a las ganancias selectivas que implica obtener dinero fácil, mayor reputación y el acceso a más compañeros sexuales, todos los cuales decrecen en sus efectos positivos para el individuo 
en los años subsiguientes. A menudo estos comportamientos son vistos como indeseables por cuanto se apartan de la conducta gregaria que la sociedad favorece y se consideran desviaciones a la norma, lo cual no necesariamente contiene toda la verdad del asunto. De hecho, la impulsividad riesgosa se presenta con mayor frecuencia en el sexo masculino (Cross, 2010). La conducta de tomar riesgos puede también entenderse como una forma de adaptación exitosa en determinadas circunstancias, en las que incluso produce efectos que incrementen la tasa de reproducción en el mediano plazo o permite acceder a más y mejores parejas, dejando atrás a los individuos más conservadores. En las sociedades arcaicas los hombres de arrojo, valentía y conducta decidida, que no temían enfrentar la inseguridad, los peligros y desafíos inesperados, salían gananciosos al obtener más propiedades, poder o prestigio. También las mejores opciones de reproducción. Salvando distancias, las colectividades modernas no se encuentran demasiado lejos de esta realidad, aunque la coreografía social resulte más sutil. Y aunque suavizadas por la cortesía y los buenos modales, el patrón masculino que reúne estas características suele estar entre los más admirados por las mujeres, con prescindencia de la cultura respectiva, como los estudios de Buss (1994) demostraron sugestivamente. De hecho, las características físicas del hombre se hallan especialmente adaptadas para simular un aspecto desafiante o permi- tirle causar un daño físico efectivo. La barba y la profundidad de la voz parecen aumentar el tamaño real y la dominancia. El tamaño, la musculatura, la agresión y la manufactura de armas incrementan directamente la habilidad para la lucha (Puts, 2010).

De todos estos comportamientos, los homicidios son probablemente los que hieren la conciencia de una manera más aguda. La agresión humana y la violencia son productos de la historia evolutiva de la especie y como tales pueden entenderse como respuestas efectivas a los desafíos que nuestros antepasados enfrentaron en sus entornos ancestrales. Estas realidades revisten muchas formas diferentes. Henson (2006) analizó la influencia ejercida por los incrementos en la población y los efectos causados por el clima en la subsecuente reducción de los recursos disponibles como dos de los motivadores básicos para la guerra en las comunidades primitivas. Shrina (2013) explica cómo las tasas de criminalidad guardan relación directa con otras variables, entre ellas la amenaza que suponen las enfermedades infecciosas. Los humanos, además de las defensas inmunológicas, hemos desarrollado otras respuestas de orden comportamental como la xenofobia dirigida contra grupos de extraños y que se exacerban significativamente cuando las condiciones de riesgo por la enfermedad aumentan. La xenofobia reduce las inhibiciones contra el daño y la explotación de los miembros que viven 
en la comunidad pero son reconocidos como externos. La segregación de esos grupos reduce la preocupación de la gente por su bienestar como minorías, al tiempo de incrementar la intolerancia y debilitar la capacidad colectiva para prevenir el delito.

La conducta violenta está asociada a una serie muy variable de pautas evolutivas, muchas de las cuales se observan también en las sociedades de primates. En ellas se distinguen patrones de competencia entre machos, ya sea por la obtención de recursos limitados destinados al forrajeo o por el acceso $\mathrm{y}$ apareamiento a las hembras, que con frecuencia resulta limitado. Ya Darwin (1871) remarcó que los machos exhiben comportamientos sustancialmente más agresivos en todas las especies de mamíferos. La competencia intrasexual de algunas variedades de primates lleva a una considerable diferencia en los índices de mortalidad de los machos si se los compara con las hembras. Kruger y Fitzgerald (2012) recuerdan que de las varias aproximaciones teóricas evolucionistas surgidas en función al problema de la criminalidad, algunas, como la del psicólogo David Buss, postulan la existencia de adaptaciones psicológicas que facilitan la producción de comportamientos homicidas para conseguir fines específicos, mientras otras, como las de Daly y Wilson, sugieren que la conducta criminal es una adaptación de otros hábitos menos letales en principio, como por ejemplo la competencia por el status, sumado a situaciones coyunturales que finalmente desembocan en asesinatos.

En el enfoque de estos dos autores (Daly y Wilson, 1988a) se remarca la importancia de la eficacia biológica o fitness para incrementar el éxito individual en la lucha cotidiana por la vida. Para comprender cómo funciona este proceso resulta imprescindible considerar el autointerés y sobre todo las percepciones que de este puedan llegar a tener los sujetos. Las personas tienden a percibir sus intereses como armoniosos con los de otros en la medida en que los factores que aumentan o disminuyen la eficacia esperada para uno tengan poco efecto directo sobre los demás. Por el contrario, los intereses se perciben como realidades en conflicto cuando las ambiciones de dos o más individuos solo pueden ser mejoradas a expensas de los intereses ajenos. La relevancia para un entendimiento de los comportamientos violentos como conducentes al homicidio, vistos como estrategias para imponer la lógica de la eficacia individual para la supervivencia, se torna así evidente. Daly y Wilson cubrieron algunas subcategorías particulares de homicidios, entre las que cuentan los infanticidios, feminicidio, parricidio y asesinatos de hombres por otros hombres surgidos de contingencias que se denominan altercados triviales. El punto clave del argumento es que la producción de crímenes varía inversamente al grado de relación ge- 
nética que existe entre el victimario y la víctima. Es decir, cuanto mayor sea la proximidad entre dos personas, menor es la posibilidad de que se produzca un homicidio entre ellos. Esta realidad debe comprenderse dentro de los lineamientos establecidos por el principio de la eficacia inclusiva, que predice un mayor flujo de recursos hacia la descendencia y los hijos así como el apoyo continuado a estos, que eventualmente podría incluir también un componente de conducta altruista.

Ciertamente que la producción de homicidios en el ámbito familiar parecería desestimar radicalmente esta conclusión, pero ello se comprende mejor cuando se toma en cuenta que la mayor parte de estas agresiones fatales ocurren entre esposos, que por supuesto no guardan una relación genética directa entre sí. Los ejemplos de muerte entre personas en una situación de consanguinidad solo alcanzan el seis por ciento del total, es decir, son bastante reducidos (Brookman, 2005). Los casos de violencia letal dirigidos hacia los hijos, si bien están presentes con determinada regularidad, muestran una incidencia considerablemente menor. Los modelos evolucionistas que tratan sobre los motivos sociales predicen que la cercanía genética se asocia de manera predominante a la mitigación del conflicto. En el caso de discordancias entre padres e hijos, el enfoque también predice que el riesgo habrá de decrecer en función de la edad, el sexo y otras características de los protagonistas. Los padres pueden mostrarse más dispuestos a incurrir en costos que van en beneficio de sus hijos cuando estos se hallan en una etapa más próxima a la madurez. En tales circunstancias también se manifiestan más reacios a utilizar tácticas que pudieran afectarlos severamente, descartando la posibilidad de producir daños duraderos o incluso la muerte. De esta manera, la probabilidad de cometer acciones criminales contra los hijos decrecen significativamente con el aumento de la edad (Daly y Wilson, 1988b).

Los homicidios más comunes nunca son planeados ni razonados, ocurren por motivos azarosos. La conducta impulsiva es un ingrediente fundamental en ellos. Pueden comenzar con discusiones circunstanciales o desentendimientos graves en aspectos sensibles como el honor, la reputación o el status entre hombres. Habitualmente van subiendo de tono hasta que comienzan a tornarse más virulentos y uno de los contendientes finalmente acaba muerto. El hecho de que los crímenes violentos resulten más frecuentes en hombres jóvenes y se cometan en perjuicio de otros del mismo sexo y que además sean con frecuencia perpetrados por personas de un nivel socioeconómico bajo en comparación con los de status social más elevado, sugieren que tienen sus raíces en la competencia entre machos y en la rivalidad reproductiva, que podría considerarse su raíz última. La universalidad de este patrón consistente en hombres en su ju- 
ventud matando a otros hombres de igual edad es uno de los supuestos que tiene la psicología evolucionista y demuestra una de las características evolutivas de la especie: el que los varones jóvenes resultan más agresivos (Marmot, 2004). Por la misma razón, la incidencia del comportamiento criminal se reduce paulatinamente con el aumento de la edad. Esa condición es un claro indicador en la declinación de la testosterona y con ella de los esfuerzos y éxito reproductivos de los miembros masculinos de la especie (Quinsey, 2002). En todas estas acciones las emociones y no la razón cumplen el rol directivo fundamental. Como afirma metafóricamente Buss (2005), las pasiones siempre otorgan el combustible motivacional para cometer un asesinato. Miller y Kanazawa (2007) estiman que esta es la razón para que la pena capital no prospere como penalidad disuasoria en los países que la aplican, pues su lógica implícita es que los homicidios siempre son planeados y premeditados. Pero la realidad es otra. La doctrina también asume que el peor castigo posible es la muerte, cuando en la lógica evolutiva hay algo aún más aborrecible, y es el total fracaso reproductivo para un hombre, que no puede competir por la consecución de parejas en los límites que impone su entorno. No debe ignorarse que la mayor ocurrencia de homicidios en los hombres, principalmente aquellos relacionados con la expresión súbita de los celos, no ocurren necesariamente porque ellos sean más propios del sexo masculino sino porque, en general, los varones cometen más acciones de este tipo que las mujeres (Campbell, 2002). El crimen y la violencia letal entre hombres, que en la tradición judeo-cristiana se origina con el incidente entre Caín y Abel, ha estado presente en las expresiones sociales de todas las culturas y mezcladas muchas veces con los rituales sagrados de estas. Persiste como una compañía constante en esta etapa más compleja de la civilización, por lo que su estudio e intelección, además de la finalidad preventiva, continuarán figurando entre las grandes prioridades de la ciencia.

\section{Conclusión}

En su corto periodo de vigencia, la psicología evolucionista cosechó tanto partidarios como detractores. Desde luego, el adoptar un enfoque con estas características respecto al comportamiento criminal necesariamente habrá de arrastrar críticas o malentendidos que a veces distorsionan sus alcances y pretensiones reales. Duntley y Buss (2004) recopilaron algunas de estas disidencias. Hay quienes sugieren que la psicología evolucionista implica que los seres humanos, al cometer actos contrarios a cuanto prescriben la legislación y las buenas costumbres, son presentados como víctimas de fuerzas ciegas e impersonales y por lo tanto se los considera como no responsables de sus actos. Por otra parte, también se ha sugerido que una explicación de estas acciones -como resultado de mecanismos psi- 
cológicos evolucionados- supone que al mismo tiempo se están justificando tales conductas, en una variedad nueva de la falacia naturalista. Sobre la inconveniencia de confundir el es con el debe ser, ya en 1739 el filósofo escocés David Hume (1711-1776) escribió cuanto había que decir en las páginas del Tratado de la naturaleza humana (1739/1984). Es un hecho que ciertas personas, cuando escuchan decir que los comportamientos humanos son dependientes de nuestra naturaleza y que estos se han formado a lo largo de incontables generaciones de individuos como respuesta a las presiones selectivas del ambiente, de inmediato asumen que tal reconocimiento significa admitir que estas son las formas de actuar que debieran darse en cualquier situación (Geher, 2006). Obviamente, la comprensión de las causas que generan una conducta asesina o violenta no significa que ella sea deseable o que no deba ser una urgencia social el erradicarlos. La ciencia también puede buscar la forma de modificar las actitudes sociales y las condiciones que propician la expresión de estas manifestaciones comportamentales para intentar suprimirlas del repertorio de los hábitos humanos, por mucho que ello cueste y a pesar del tiempo que pueda demandar.

Sin abandonar del todo el enfoque evolucionista, algunos autores optan por una explicación del comportamiento criminal que combina diferentes marcos explicativos, señalando las ventajas y desventajas de cada uno, así como las posibles limitaciones que surgen al ser utilizados como esquemas interpretativos individuales. Brannigan (1997), por ejemplo, emplea aspectos de la teoría general del crimen, en especial el autocontrol, la teoría del poder del control y el enfoque del ciclo vital combinados con la aproximación de Daly y Wilson (1988a, 1988b, 2001). Encuentra que una aplicación conjunta de los tres modelos es capaz de generar una alternativa conceptual más robusta. Pero también señala algunas limitaciones en el modelo evolucionista, como la yuxtaposición entre los varios mecanismos psicológicos propuestos para el análisis del crimen, una aproximación que considera incorrecta para los procesos del desarrollo humano y la socialización que inciden sobre los homicidios y la normatividad de la conducta criminal que presuntamente favorecen las teorías darwinianas. Este es el mismo punto ya discutido al recordar los argumentos de Duntley y Buss (2004) un poco antes. De hecho, y como cualquier aportación científica, la psicología evolucionista es un proyecto coherente que habrá de recibir correcciones y mayores refinamientos en el futuro, sin necesidad de estigmatizarla, como una psicología mal adaptada (Richardson, 2007). No pretende exclusividad ni hegemonía teórica alguna. Pero es una herramienta poderosa que exhibe muchas posibilidades de comprensión fundamentada del comportamiento humano y cuyas potencialidades de aplicación ya co- 
mienzan a perfilarse en algunas publicaciones recientes (Roberts, 2012). Lo importante como estrategia de investigación es que centra sus fortalezas en el reconocimiento de la naturaleza, esencialmente adaptativa del comportamiento humano y en la admisión de que este no es atemporal sino que responde a las mismas exigencias impuestas por el ambiente a todos y cada uno de los miembros de la especie. Es así como el antiguo problema de la criminalidad, presente en las reflexiones psicológicas desde mucho antes de la adopción del método experimental y convidado obligado en las discusiones sobre las políticas relacionadas con el orden social, recibe nuevas luces para arribar a una comprensión realista, efectiva y contrastable de sus verdaderas causas.

\section{ReferenCiAs}

Alcock, J. (1989). Animal behavior. An evolutionary approach. Sunderland: Sinauer.

Anthony, T., y Anthony, D. (2008). Psychologizing criminals and the Frankfurt School's critique. En T. Anthony y C. Cunneen (Eds.), The critical criminology companion (pp. 43-54). Sidney: Hawkins Press.

Baldwin, J. M. (1895). Mental development in the child and the race. New York: Macmillan.

Brannigan, A. (1997). Self control, social control, and evolutionary psycholo- gy: towards an integrated perspective on crime. Canadian Journal of Criminology, 39(4), 403-431.

Brookman, F. (2005). Understanding homicide. New Delhi: Sage.

Brown, S. E., Esbensen, F. A., y Geis, G. (2013). Criminology. Explaining crime \& its context (8. ${ }^{\mathrm{a}}$ ed.). Waltham: Andersen Publishing.

Buss, D. M. (1994). The evolution of desire. Strategies of human mating. New York: Basic Books.

Buss, D. M. (2005). The murderer next door. Why the mind is designed to kill. New York: Penguin.

Buss, D. M. (2012). The evolutionary psychology of crime. Journal of Theoretical and Philosophical Criminology, 1(1), 90-98.

Buss, D. M., y Duntley, J. D. (2006). The evolution of aggression. En M. Schaller, J. A. Simpson y D. T. Kenrick (Eds.), Evolution and social psychology (pp. 263-285). New York: Psychology Press.

Cahan, E. D. (1984). The genetic psychologies of James Mark Baldwin and Jean Piaget. Developmental Psychology, 20(1), 128-135.

Campbell, A. (2002). A mind of her own. The evolutionary psychology of women. Oxford: Oxford University Press.

Cartwright, J. (2000). Evolution and human behavior. Darwinian perspectives on human nature. Massachusetts: The MIT Press. 
Cross, C. P. (2010). Sex differences in same-sex direct aggression and sociosexuality: The role of risky impulsivity. Evolutionary Psychology, 8(4), 779-772.

Daly, M., y Wilson, M. (1988a). Homicide. News Brunswick: Transaction.

Daly, M., y Wilson, M. (1988b). Evolutionary social psychology and family homicide. Science, 242, 519-524.

Daly, M. y Wilson, M. (2001). Risktaking, intrasexual competition, and homicide. Nebraska Symposium on Motivation, 47, 1-36.

Darwin, C. (1859). On the origin of species by means of natural selection, or the preservation of favoured races in the struggle for life. London: John Murray.

Darwin, C. (1871). The descent of man and selection in relation to sex. New York: D. s. Appleton and Company.

Darwin, C. (1872). The expression of the emotions in man and animals. London: John Murray.

Duntley, J. D. y Buss, D. M. (2004). The evolution of evil. En A. G. Miller (Ed.), The social psychology of good and evil (pp. 102-123). New York: The Guilford Press.

Duntley, J. D. y Buss, D. M. (2012). The evolution of stalking. Sex roles, 66, 311-327.

Duntley, J. D. \& Shackelford, T. K. (Eds.) (2008). Evolutionary forensic psychology. New York: Oxford University Press.
Durkheim, E. (1919). Les règles de la méthode sociologique. Paris: Librairie Félix Alcan.

Figueredo, A. J., Gladden, P. R., y Hohman, Z. (2012). The evolutionary psychology of criminal behaviour. En S. Craig Roberts (Ed.), Applied Evolutionary Psychology (pp. 201-221). New York: Oxford University Press.

Francis, K. A. (2007). Charles Darwin and the origin of species. Westport: Greenwood Press.

Geary, D. C. (2005). Evolution of paternal investment. En D. M. Buss (Ed.), The handbook of evolutionary psychology (pp. 483-505). Hoboken: Wiley.

Geher, G. (2006). Evolutionary psychology is not evil! (... and here's why ...). Psychological Topics, 15(2), 181-202.

Hall, B. K. (1999). Evolutionary developmental biology (2. ${ }^{a}$ ed.). Dordrecht: Kluwer Academic Publishers.

Haskins, R. W. (1839). History and progress of phrenology. Buffalo: Steele \& Peck.

Henson, H. K. (2006). Evolutionary psychology, memes and the origin of war. Mankind Quarterly, 46(4), 443-459.

Hergenhahn, B. R. y Henley, T. B. (2013). An introduction to the history of psychology (7. a ed.). Boston: Cengage Learning.

Hume, D. (1739/1984). Tratado de la naturaleza humana. Buenos Aires: Orbis. 
James, W. (1890). The principles of psychology. New York: Henry Holt and Company.

Kinner, S. (2003). Psychopathy as an adaptation. Implications for society and social policy. En R. W. Bloom y N. Dess (Eds.), Evolutionary psychology and violence. A primer for policymakers and public policy advocates (pp. 57-82). Westport: Praeger.

Kruger, D. J. y Fitzgerald, C. J. (2012). Evolutionary perspectives on malemale competition, violence, and homicide. En T. K. Shackelford y V. Weekes-Shackelford (Eds.), The Oxford handbook of evolutionary perspectives on violence, homicide, and war (pp. 153-170). New York: Oxford University Press.

Lombroso, C. (1876). L'uomo delinquent studiato in rapporto alla antropologia, alla medicina legale ed alle discipline carcerarie. Milano: Ulrico Hoepli Libraio-Editore.

Marmot, M. (2004). The status syndrome. How social standing affects our health and longevity. New York: Henry Holt \& Company.

McDougall, W. (1912). An introduction to social psychology (6. ${ }^{\mathrm{a}}$ ed.). London: Methuen.

McKibbin, W. F., Shackelford, T. K., Goetz, A. T. y Starratt, V. G. (2008). Why do men rape? An evolutionary psychological perspective. Review of General Psychology, 12(1), 86-97.
Miller, A. S. y Kanazawa, S. (2007). Why beautiful people have more daughters: From dating, shopping, and praying to going to war and becoming a billionaire - Two evolutionary psychologists explain why we do what we $d o$. New York: Pedigree.

Mishra, S. y Lalumière, M. L. (2008). Risk-taking, antisocial behavior, and life histories. En J. Duntley y T. K. Shackelford (Eds.), Evolutionary forensic psychology (pp. 139-159). New York: Oxford University Press.

Morgan, C. L. (1896). An introduction to comparative psychology. London: Walter Scott, Ltd.

Padhy, P. (2006). Crime and criminology. Volume 3. Criminological Theories. Delhi: Isha Books.

Passingham, R. y Wise, S. (2012). The neurobiology of the prefrontal cortex. Anatomy, evolution and the origin of insight. New York: Oxford University Press.

Puts, D. A. (2010). Beauty and the beast: Mechanism of sexual selection in humans. Evolution and Human Behavior, 31, 157-175.

Quinsey, V. L. (2002). Evolutionary theory and criminal behavior. Legal and Criminological Psychology, 7, 1-13.

Quinsey, V. L. (2010). The evolutionary psychology perspective. En J. M. Brown y E. A. Campbell (Eds.), The Cambridge handbook of forensic psychology (pp. 43-48). New York: Cambridge University Press. 
Raff, R. A. (1996). The shape of life. Genes, development, and the evolution of animal form. Chicago: The University of Chicago Press.

Rafter, N. (2008). The criminal brain. Understanding biological theories of crime. New York: New York University Press.

Renneville, M. (2000). Le langage des crânes. Une histoire de la phrénologie. Paris: Institut d'édition Sanofi-Synthélabo.

Renneville, M. (2006). The French Revolution and the origins of French Criminology. En P. Becker y R. F. Wetzell. Criminals and their scientists. The history of criminology in international perspective (pp. 25-41). New York: Cambridge University Press.

Richards, R. J. (1989). Darwin and the emergence of evolutionary theories of mind and behavior. Chicago: The University of Chicago Press.

Richardson, R. C. (2007). Evolutionary Psychology as Maladapted Psychology. Cambridge, Massachusetts: The MIT Press.

Roberts. S. C. (Ed.) (2012). Applied Evolutionary Psychology. New York: Oxford University Press.

Röell, D. R. (2000). The world of instinct. Assen: Van Gorcum \& Comp.

Romanes, G. J. (1884). Animal intelligence. New York: D. Appleton and Company.
Ruse, M. (2001). The evolution wars: A guide to the debates. New Brunswick: Rutgers University Press.

Sapp, J. (2003). Genesis: The evolution of biology. New York: Oxford University Press.

Schmitt, D. P. (2010). Human mate choice. En M. P. Muehlenbein (Ed.), Human evolutionary biology (pp. 295-308). New York: Cambridge University Press.

Schultz, D. P., y Schultz, S. E. (2011). A history of modern psychology (10. ${ }^{\mathrm{a}}$ ed.). Belmont: Wadsworth, Cengage Learning.

Shanahan, T. (2004). The evolution of darwinism: selection, adaptation and progress in evolutionary biology. Cambridge: Cambridge University Press.

Shiraev, E. (2014). A history of psychology. A global perspective (2. ${ }^{\mathrm{a}}$ ed.). London: Sage.

Shrina, I. (2013). Guns, germs, and stealing: Exploring the link between infectious disease and crime. Evolutionary Psychology, 11(1), 270-287.

Stefoff, R. (1996). Charles Darwin and the evolution revolution. New York: Oxford University Press.

Sutherland, E. H., Cressey, D. R., y Luckenbill, D. F. (1992). Principles of criminology. Lanham: General Hall.

Tolman, C. W. (1987). Theories of mental evolution in comparative psycholo- 
gy: Darwin to Watson. En E. Tobach (Ed.), Historical perspectives and the international status of Comparative psychology (pp. 15-23). Hillsdale: Lawrence Erlbaum Associates.

Tooby, J., y Cosmides L. (1992). The psychological foundations of culture. En J. H. Barkow, L. Cosmides, y J. Tooby (Eds.), The adapted mind. Psychology and the generation of culture (pp. 19-136). New York: Oxford University Press.

Tooby, J., y Cosmides, L. (2005). Conceptual foundations of evolutionary psychology. En D. M. Buss (Ed.), The handbook of evolutionary psychology (pp. 5-67). Hoboken: Wiley.
Vallejo, M. (2009). Paradojas y éxitos de una empresa imposible. Análisis de Traité philosophique et physiologique de L'hérédité naturelle (18471850) de Prosper Lucas. Anuario de Investigaciones, 16, 207-215.

Walklate, S. (2007). Understanding criminology. Current theoretical debates. Berkshire: Open University Press.

Wilson, E. O. (2000). Sociobiology: The new synthesis. Cambridge MA: Harvard University Press. Edición por el 25. ${ }^{\circ}$ aniversario.

Wortley, R. (2011). Psychological criminology: an integrative approach. Abingdon: Routledge. 